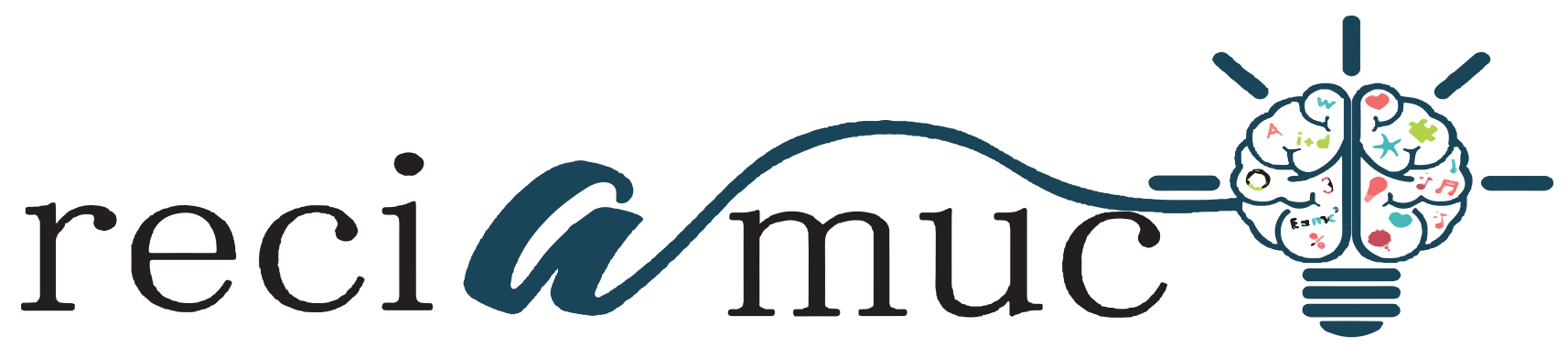

DOI: $10.26820 /$ reciamuc/4.(1).enero.2020.29-39

URL: https://reciamuc.com/index.php/RECIAMUC/article/view/425

EDITORIAL: Saberes del Conocimiento

REVISTA: RECIAMUC

ISSN: $2588-0748$

TIPO DE INVESTIGACIÓN: Artículo de Revisión

CÓDIGO UNESCO: 3205 Medicina Interna

PAGINAS: 29-39

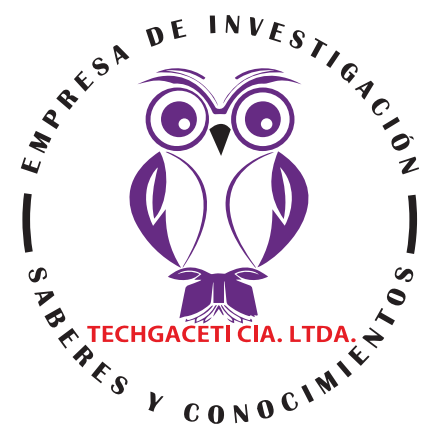

\title{
Turbinectomia en hipertrofia de cornetes nasales
}

\section{Turbinectomy in hypertrophy of nasal turbinates Turbinectomia na hipertrofia de cornetos nasais}
Isis Natali Parrales Vidal'; Katherine Tatiana Reyes Tigrero²; María Verónica Crespo Zamora3; Juan Gabriel Chavez Reyes ${ }^{4}$

RECIBIDO: 18/11/2019 ACEPTADO: 20/12/2019 PUBLICADO: 31/01/2020

1. Médico; Investigadora Independiente; Guayaquil, Ecuador; isisparrales_@hotmail.com; (D) https://orcid. org/0000-0002-5235-1350

2. Médico; Investigadora Independiente; Guayaquil, Ecuador; katreyes16@gmail.com; (D) https://orcid.org/00000001-7387-1219

3. Médico; Investigadora Independiente; Guayaquil, Ecuador; veronicacrespoz29@outlook.com; (D) https://orcid. org/0000-0002-4098-7618

4. Médico; Investigadora Independiente; Guayaquil, Ecuador; juangabrielchavez@gmail.com; (D) https://orcid. org/0000-0002-2986-3710

CORRESPONDENCIA

Isis Natali Parrales Vidal

isisparrales_@hotmail.com

Guayaquil, Ecuador 


\section{RESUMEN}

En la ciencia médica a nivel internacional no existe una característica específica que defina que un paciente tenga que ser sometido a una cirugía de cornetes. Este tipo de cirugía se centra más que todo en aquellas personas que presentan quejas ante el especialista de acuerdo a una obstrucción nasal, lo que conlleva a la realización del examen clínico por parte del cirujano. Aunque la mayoría quirúrgica, las técnicas empleadas para tratar esta patología, demuestran una mejora subjetiva y objetiva en el flujo de aire nasal postoperatorio y las puntuaciones subjetivas, sin embargo, es difícil decir qué técnica es la más adecuada para tratar la ampliación del cornete inferior, no obstante, durante el desarrollo del proceso investigativo se observa, que la reducción del cornete inferior para nasal y los síntomas obstructivos causados por los cornetes agrandados son un procedimiento útil y están reservados para pacientes que no responden al tratamiento médico o en quien el manejo médico está contraindicado.

Palabras clave: Hipertrofia de Cornetes, Obstrucción Nasal, Radiofrecuencia, Láser, Turbinectomía.

\section{ABSTRACT}

In medical science at the international level there is no specific characteristic that defines that a patient has to undergo surgery of turbinates. This type of surgery focuses mostly on those people who complain to the specialist according to a nasal obstruction, which leads to the clinical examination by the surgeon. Although the surgical majority, the techniques used to treat this pathology, demonstrate a subjective and objective improvement in the postoperative nasal air flow and subjective scores, however, it is difficult to say which technique is the most appropriate to treat the enlargement of the inferior turbinate However, during the development of the investigative process, it is observed that the reduction of the inferior nasal turbinate and the obstructive symptoms caused by the enlarged turbinates are a useful procedure and are reserved for patients who do not respond to medical treatment or in whom the management Doctor is contraindicated.

Keywords: Horn hypertrophy, Nasal Obstruction, Radiofrequency, Laser, Turbinectomy.

\section{RESUMO}

Na ciência médica em nível internacional, não há característica específica que defina que um paciente tenha que ser submetido à cirurgia de cornetos. Esse tipo de cirurgia se concentra principalmente nas pessoas que reclamam com o especialista de acordo com uma obstrução nasal, o que leva ao exame clínico do cirurgião. Embora a maioria cirúrgica, as técnicas utilizadas para tratar essa patologia, demonstrem uma melhora subjetiva e objetiva no fluxo aéreo nasal e nos escores subjetivos no pós-operatório, é difícil dizer qual técnica é a mais adequada para tratar o aumento do corneto inferior. No entanto, durante o desenvolvimento do processo investigativo, observa-se que a redução do corneto nasal inferior e os sintomas obstrutivos causados pelos cornetos aumentados são um procedimento útil e reservado a pacientes que não respondem ao tratamento médico ou nos quais o manejo Médico é contra-indicado.

Palavras-chave: Hipertrofia do chifre, Obstrução nasal, Radiofreqüência, Laser, Turbinectomia. 


\section{Introducción}

La obstrucción crónica de la vía aérea nasal es una los síntomas más frecuentes encontrados por el otorrinolaringólogo. La mayoría de estos pacientes sufren de cornetes inferiores hipertrofiados. Está bien establecido que los cornetes nasales representan el control de flujo de aire nasal primario por lo que tienen un papel significativo en el mantenimiento de la salud normal de las vías respiratorias y esto se hace principalmente por su mucosa. (Steiner W., 2006.)

Esta importante parte anatómica de la nariz contribuye a filtración de aire, humidificación de aire y también a calentamiento del aire inhalado (Leong \& Eccles, 2015). Aunque esta condición no es una amenaza para la vida, la rinitis crónica puede tener un gran impacto en la calidad de vida de los pacientes por lo que es necesario establecer rápidamente un tratamiento adecuado.

Los médicos disponen de muchas técnicas para curar los cornetes agrandados. Los autores (Hol \& Huizing, 2016) en su estudio expresan que la mucosa nasal de los cornetes se hincha como parte del ciclo nasal, pero esta inflamación fisiológica puede agravarse por inhalación de alérgenos, infecciones, irritantes en el aire, entre otros, en ese caso, el médico le receta tratamientos al paciente como aerosoles nasales salinos, corticosteroides tópicos aerosoles, antihistamínicos, agentes simpaticomiméticos, fármacos anticolinérgicos, solución de nitrato de plata, varios inmunoestimulantes y capsaicina como terapia de terapia de primera línea.

Sin embargo, estos tratamientos podrían ser inadecuados para lograr una resolución significativa de la obstrucción nasal. Es por eso que los pacientes cuya condición es resistente a la terapia médica, el paciente, puede buscar tratamiento adicional. En estos casos, se recomienda la reducción quirúrgica del cornete inferior hipertrófico teniendo en consideración el fracaso de la terapia médica como tratamiento inicial.

El autor (Mucci S., 2017) afirma que la cirugía turbinada es un procedimiento quirúrgico que consiste en la reducción volumétrica del cornete inferior y por lo tanto, disminuye la resistencia de las vías respiratorias mejorando la respiración nasal.

En ocasiones tanto la porción ósea como la mucosa del cornete pueden ser hipertrófico. Generalmente a pacientes con alergia, se le asocia rinitis con hinchazón de la mucosa. Por otro lado, la ampliación unilateral de la estructura del hueso se observa en pacientes con desviación septal grave. (Mucci S., 2017)

Por eso es muy importante encontrar la causa real de la obstrucción nasal para que el cirujano pueda elegir las técnicas quirúrgicas adecuadas. La reducción de los cornetes inferiores agrandados puede lograrse con una multitud de diferentes técnicas quirúrgicas, como la turbinoplastia (resección submucosa outfracture), ablación por radiofrecuencia (RFA), extra-electrocauterización mucosa o submucosa, turbinectomía, resección o ablación asistida por láser, crioterapia, ultrasonido reducción. (Passali, 2013)

\section{Métodos y materiales}

Para el desarrollo de este proceso investigativo, se plantea como metodología la encaminada hacia una orientación científica particular que se encuentra determinada por la necesidad de indagar en forma precisa y coherente una situación, en tal sentido (Davila, 2015) define la metodología "como aquellos pasos previos que son seleccionados por el investigador para lograr resultados favorables que le ayuden a plantear nuevas ideas".(p.66)

Lo citado por el autor, lleva a entender que el desarrollo de la acción investigativa busca simplemente coordinar acciones enmarcadas en una revisión bibliográfica con el fin de complementar ideas previas relacio- 
nadas Turbinectomia en hipertrofia de cornetes nasales a través de una revisión de literatura, para así finalmente elaborar un cuerpo de consideraciones generales que ayuden a ampliar el interés propuesto.

\section{Tipo de Investigación}

Dentro de toda práctica investigativa, se precisan acciones de carácter metodológico mediante las cuales, se logra conocer y proyectar los eventos posibles que la determinan, así como las características que hacen del acto científico un proceso interactivo ajustado a una realidad posible de ser interpretada. En este sentido, se puede decir, que la presente investigación corresponde al tipo documental, definido por Castro (2016), "se ocupa del estudio de problemas planteados a nivel teórico, la información requerida para abordarlos se encuentra básicamente en materiales impresos, audiovisuales y /o electrónicos". (p.41).

En consideración a esta definición, la orientación metodológica permitió la oportunidad de cumplir con una serie de actividades inherentes a la revisión y lectura de diversos documentos donde se encontraron ideas explicitas relacionadas con los tópicos encargados de identificar a cada característica insertada en el estudio. Por lo tanto, se realizaron continuas interpretaciones con el claro propósito de revisar aquellas apreciaciones o investigaciones propuestas por diferentes investigadores relacionadas con el tema de interés, para luego dar la respectiva argumentación a los planteamientos, en función a las necesidades encontradas en la indagación.

\section{Fuentes Documentales}

El análisis correspondiente a las características que predomina en el tema seleccionado, llevan a incluir diferentes fuentes documentales encargadas de darle el respectivo apoyo y en ese sentido cumplir con la valoración de los hechos a fin de generar nuevos criterios que sirven de referencia a otros procesos investigativos. Para
(CASTRO, 2016) las fuentes documentales incorporadas en la investigación documental o bibliográfica, "representa la suma de materiales sistemáticos que son revisados en forma rigurosa y profunda para llegar a un análisis del fenómeno".(p.41). Por lo tanto, se procedió a cumplir con la realización de una lectura previa determinada para encontrar aquellos aspectos estrechamente vinculados con el tema, con el fin de explicar mediante un desarrollo las respectivas apreciaciones generales de importancia.

\section{Técnicas para la Recolección de la Infor- mación}

La conducción de la investigación para ser realizada en función a las particularidades que determinan a los estudios documentales, tiene como fin el desarrollo de un conjunto de acciones encargadas de llevar a la selección de técnicas estrechamente vinculadas con las características del estudio. En tal sentido, (Bolívar, 2015), refiere, que es "una técnica particular para aportar ayuda a los procedimientos de selección de las ideas primarias y secundarias". (p. 71).

Por ello, se procedió a la utilización del subrayado, resúmenes, fichaje, como parte básica para la revisión y selección de los documentos que presentan el contenido teórico. Es decir, que mediante la aplicación de estas técnicas se pudo llegar a recoger informaciones en cuanto a la revisión bibliográfica de los diversos elementos encargados de orientar el proceso de investigación. Tal como lo expresa, (Bolívar, 2015) "las técnicas documentales proporcionan las herramientas esenciales y determinantes para responder a los objetivos formulados y llegar a resultados efectivos" (p. 58). Es decir, para responder con eficiencia a las necesidades investigativas, se introdujeron como técnica de recolección el método inductivo, que hizo posible llevar a cabo una valoración de los hechos de forma particular para llegar a la explicación desde una visión general. 
Asimismo, se emplearon las técnicas de análisis de información para la realización de la investigación que fue ejecutada bajo la dinámica de aplicar diversos elementos encargados de determinar el camino a recorrer por el estudio, según, (Bolívar, 2015) las técnicas de procesamiento de datos en los estudios documentales "son las encargadas de ofrecer al investigador la visión o pasos que debe cumplir durante su ejercicio, cada una de ellas debe estar en correspondencia con el nivel a emplear" (p. 123). Esto indica, que para llevar a cabo el procesamiento de los datos obtenidos una vez aplicado las técnicas seleccionadas, tales como: fichas de resumen, textual, registros descriptivos entre otros, los mismos se deben ajustar al nivel que ha sido seleccionado.

\section{Resultados}

Hay tres cornetes emparejados: superior, medio e inferior: ubicado en la pared nasal lateral. Estos huesos son pequeños, delgados y tienen forma de concha. Están estructurados de la siguiente manera: el superior y los cornetes medios son parte del hueso etmoides, mientras que los cornetes inferiores forman uno separado. Entre los cornetes y la pared nasal lateral, existe un espacio llamado meato.

El cornete superior, que está cubierto por las vías respiratorias y olfativas (epitelio), se puede encontrar en la parte alta en la bóveda nasal y generalmente crece de la placa cribiforme del hueso etmoidal. El cornete medio es una extensión ósea del etmoides, se caracteriza por una amplia variación en origen, en algunos casos derivados de la cribosa placa, la lámina papirácea o el uncinado proceso. El hueso cornete inferior tiene su origen en la porción inferior de la pared nasal lateral. El hueso mismo es penetrado fuertemente por los canales vasculares, que abastecen el epitelio respiratorio suprayacente. (Archer \& Meyers, 2019)
El conducto lagrimal sale a la nariz debajo de la parte inferior de la porción anterior de esta estructura. El inferior el cornete está formado por una capa central de hueso cubierto, en cada vista, con una capa mucosa. La ampliación de los cornetes se atribuye comúnmente a dilatación de los senos venosos submucosos debido a congestión con sangre. (Farmer \& Eccles, 2012)

Los términos "hipertrofia" e "hiperplasia" se han usado indistintamente para la descripción de la ampliación del cornete. Sin embargo, "Hipertrofia" se debe utilizar para la ampliación del cornete resultante de un aumento en tamaño de sus células, mientras que "hiperplasia" es un agrandamiento debido a un aumento en el número de células.

En un estudio realizado por (Gindros \& Kantas, 2009) acerca de un caso de rinitis antes de ser sometido a cirugía, las imágenes revelaron metaplasia severa de la epitelio nasal, estratificación evidente en el epitelio e hiperplasia, degeneración de células epiteliales, pérdida de cilios e interrupción de las conexiones intercelulares, tal cual se aprecia en la figura 1. 


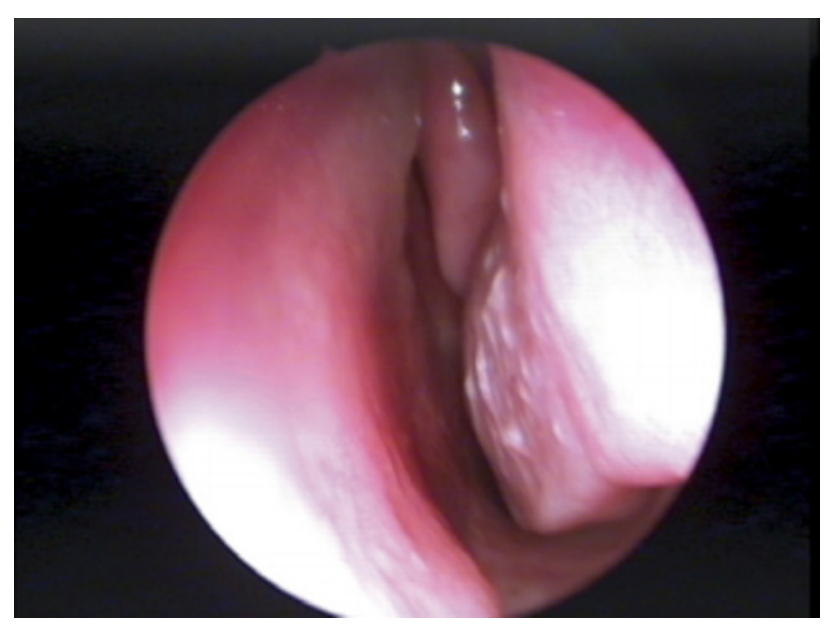

Figura 1. Hipertrofia de la mucosa del cornete inferior - examen endoscópico nasal

Fuente: (Gindros \& Kantas, 2009)

Además, aumento del grosor de la membrana basilar, edema, sobreproducción de moco nasal e inflamatorio. Adicionalmente se observó infiltración en la lámina propia, mayor número de células caliciformes y submucosas y las aperturas del numero de glándulas también se incrementaron.

\section{Cirugía turbinada}

A pesar de la popularidad de la cirugía de cornetes, los otorrinolaringólogos no tienen una manera uniforme y estandarizada para definir hipertrofia de cornetes o para seleccionar un paciente para cirugía de cornetes. Además, no existe una forma de seleccionar el tipo de cirugía de cornete necesaria. Esto sigue siendo un juicio clínico basado en el paciente en los síntomas, en el examen ENT, la rinomanometría y los hallazgos endoscópicos nasales.

\section{Técnicas quirúrgicas}

\section{Turbinectomía}

La turbinectomía es una maniobra quirúrgica que consiste en la resección parcial o completa del cornete inferior, realizado con las tijeras o el microdebrider. Este tipo de procedimiento se puede realizar con y sin el uso de un endoscopio nasal y consiste en la eliminación del tejido blando del aspecto lateral del cornete inferior y el hueso sub- yacente, cuando la hipertrofia ósea también está involucrada. (Ozcan, Gedikli, Ozcan, Pasaoglu, \& Dere, 2008)

Como resultado de la turbinectomía parcial o total, se tiene una mejora importante de la respiración nasal. Sin embargo, algunos efectos secundarios pueden ser observados después de este procedimiento. E crudo expuesto los bordes y el hueso de la mucosa pueden conducir a la formación de costras nasales con necesidad de desbridamiento postoperatorio. A pesar del control de electrocauterio, los bordes de la mucosa pueden continuar sangrando y puede ser necesario el taponamiento nasal para hemostasia. (Zapanta, 2014)

A veces, el dolor postoperatorio es muy importante. La rinitis atrófica también es una amenaza para los pacientes sometido a estos procedimientos (Ophir D., 2015). Alguna evidencia existe que la turbinectomía puede conducir a una alta incidencia del síndrome de nariz vacía, un síndrome iatrogénico de insuficiencia nasal crónica debido a cirugía nasal prolongada. La resección excesiva genera atrofia, costras, sangrado, lo que produce que la respiración nasal está deteriorada y la calidad de vida del paciente se ve afectada. (Passali, 2013) 


\section{Turbinoplastia}

Se pueden colocar dos procedimientos diferentes en la categoría de turbinoplastia:

resección submucosa y fractura inferior de cornete. En caso de una técnica submucosa de resección, se realiza una incisión en el cabeza del cornete. Parte del tejido de cornete es extirpado con la ayuda de un microdebrider y disector, permitiendo que la mucosa se recupere en un tamaño más pequeño de hueso corneado. Como resultado puede ocurrir una perforación en la mucosa o el cirujano tener una aproximación imprecisa del corte, costras, sinequias y sangrado.

La fractura externa es otra técnica quirúrgica, quizás la forma más sencilla de realizar una cirugía para la obstrucción nasal. Consiste en fracturar el hueso de la parte inferior turbinar y empujarlo lateralmente, manteniendo posteriormente esta posición con la ayuda de un taponamiento nasal (Goode, 2010).

Después de múltiples estudios que comparan diferentes tipos de reducción de cornete, los resultados han demostrado que el más alto grado de función nasal normal con restauración de obtuvo aclaramiento mucociliar y secreción de IgA después de la resección submucosa, los resultados siguen siendo lo mejor después de 6 años. La fractura del inferior turbinado realizado después de la resección submucosa del cornete demostró mejorar los resultados a largo plazo. (Leong \& Eccles, 2015)

\section{Electrocauterización}

En el pasado, la electrocauterización de la parte inferior del cornete ha sido el procedimiento más frecuente realizado para reducir el volumen del turbinado inferior, la ablación térmica superficial o submucosa produce cicatrices, fibrosis y obliteración de la senos venosos El concepto clásico establece el hecho de que la electrocauterización será efectiva si el cornete es capaz de descon- gestionarse. (Jones, 2013)

Los estudios han mostrado buenos resultados en el $78 \%$ de los casos a las 2 semanas del postoperatorio y en el $76 \%$ de los casos a los 2 meses después del procedimiento. En su estudio, (Fradis, Golz, \& Danino, 2011) concluyeron que, dos meses después de la operación, el $70.3 \%$ de los pacientes experimentado mejora subjetiva en la respiración nasal, mientras que el $80.2 \%$ tenía buena respiración nasal.

Una importante ventaja de la diatermia submucosa está representada por el hecho de que se puede hacer bajo anestesia local como procedimiento de ambulatorio, siendo seguro y efectivo tanto a corto como a largo plazo.

En un análisis histopatológico postoperatorio de la nariz realizado por (Chhabra, 2009) la mucosa reveló una disminución del edema intercelular. Sin embargo, la regeneración de los cilios en la mucosa turbinada no se observó; no obstante se observó una ligera reducción de moco, una sobreproducción de colágeno y el epitelio respiratorio fue degenerado aplanado estratificado.

\section{Láser}

Varios sistemas láser han sido probados para turbinar reducción, pero la mayoría de la experiencia se ha ganado con el dióxido de carbono (CO2), argón, diodo, neodimio-itrio

granate de aluminio ( $\mathrm{Nd}$ : YAG), fosfato de titanio-potasio (KTP), holmio: granate de itrio-aluminio (Ho: YAG) láser, láser magnético. Diferentes tipos de láseres pueden usarse en un contacto o sin contacto modo (Passali, 2013). La principal ventaja de usar un rayo láser para la cirugía reductiva del cornete inferior reside en el hecho de que el láser no produce daños en el entorno tejidos, aparte del área que requiere tratamiento.

Por ejemplo, para el uso de Nd: YAG y diodo el láser (Figura 2) está indicado si la inflama-

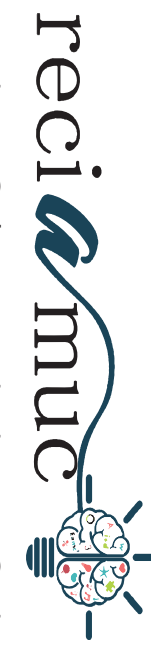


ción de la mucosa afecta todo el cornete. Con una fibra láser flexible, guiada por un rinoscopio láser, incluso la parte posterior del cornete inferior puede ser tratado. En contraste, porque de su haz recto, el láser de CO2 es especialmente adecuado para reducir la punta hiperplástica del cornete.
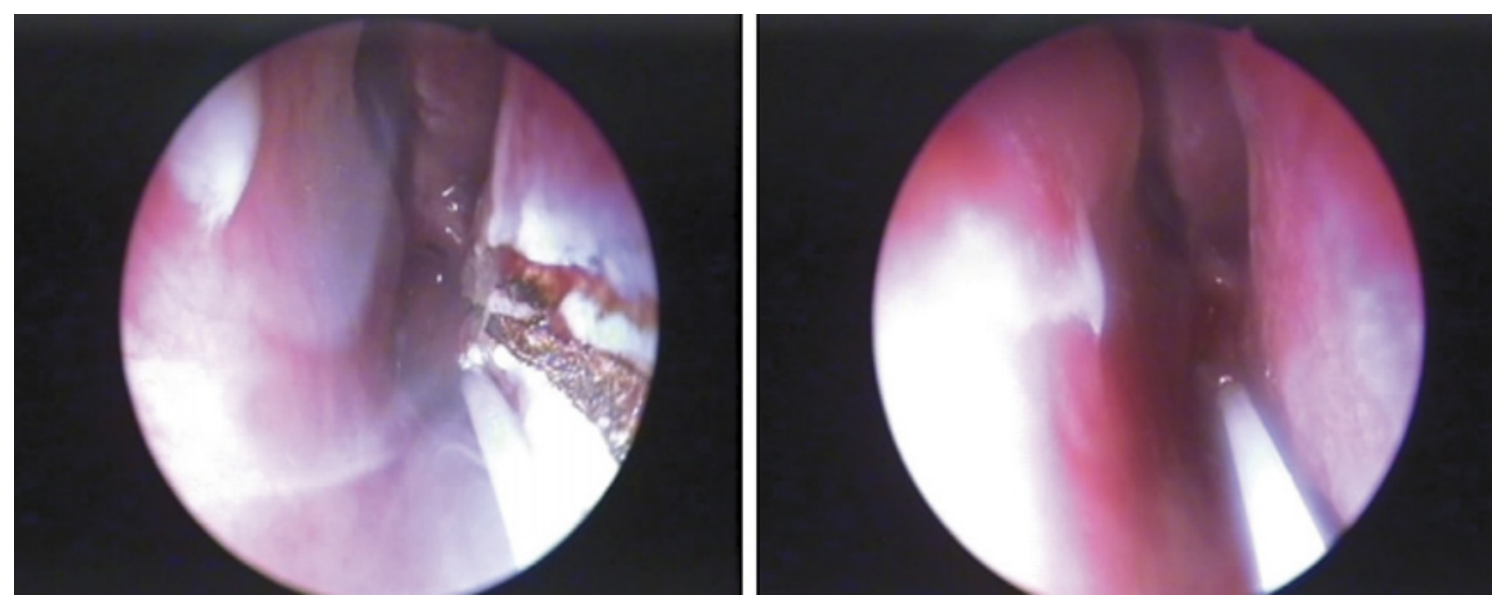

Figura 2. Vista intraoperatoria: reducción con láser de diodo de la mucosa del cornete inferior

Fuente: (Passali, 2013)

Diversas técnicas para reducir los cornetes con se han descrito con el uso del láser. Las investigaciones han mostrado que la técnica de punto único es la más adecuada para el láser de CO2. En esta técnica, la inflamación de la punta del cornete se trata con puntos láser único, lo que induce contracción de la mucosa con cicatrización posterior. En contraste con el láser de $\mathrm{CO} 2$, el tratamiento de cornete con otro tipo de los láseres, como el diodo, Nd: YAG, se llevan a cabo aplicando una técnica de contacto o sin contacto. Lo inmediato del efecto de la luz láser debe ser apenas reconocible como una pequeña mancha que muestra palidez de la mucosa. La luz láser penetra en los tejidos hasta una profundidad de $10 \mathrm{~mm}$ y se absorbe en el cuerpo cavernoso de la red submucosa vascular del cornete. Esto lleva a una vasculitis seguida de cicatrización prolongada con contracción y reducción secundaria del potencial de los cornetes para hinchazón. (Steiner W., 2006.)

La cirugía se realiza como un procedimiento ambulatorio, bajo anestesia local. La cavidad nasal es secuencialmente anestesiado, primero con lidocaína al 10\% spray nasal, seguido de prendas de algodón empapadas en lidocaína / mezcla de nafazolina colocada en ambas fosas nasales durante 15 minutos. (Steiner W., 2006.). Después de la cirugía, quedan las cavidades nasales desempaquetadas. Se aconseja a los pacientes que no se suenen la nariz durante 24 horas para prevenir un sangrado nasal. Además, todos los pacientes están informados para esperar aproximadamente 7-10 días de mayor congestión nasal y rinorrea debido a hinchazón de la mucosa intranasal.

Por lo tanto, lo claramente beneficioso el efecto sobre la respiración nasal generalmente no es evidente hasta varias semanas después. Esto puede explicarse por la regresión gradual del edema mucoso reactivo y el proceso de cicatrización submucosa que comienza lentamente, que tarda entre 4 y 6 meses en completarse.

Estudios histopatológicos mostraron una reducción en el número y la función de las células de la glándula (Elwany \& Abel, 2009), con una disminución del número de vasos y 
glándulas nasales. Debido a estos efectos, el reflejo secretor se cambia y el resultado es una reducción de la rinorrea.

\section{Cirugía de radiofrecuencia}

La radiofrecuencia (RF) es un método quirúrgico que puede ser usado de manera segura para la reducción del cornete inferior (Passali, 2013). La energía de la RF se usa para determinar una lesión de tejido submucoso, que conduce a la reducción del volumen del tejido. La sonda térmica utilizada en esta técnica particular es introducido en el cornete inferior y la radioenergía es liberada para así cortar parte del tejido. (Zapanta, 2014)

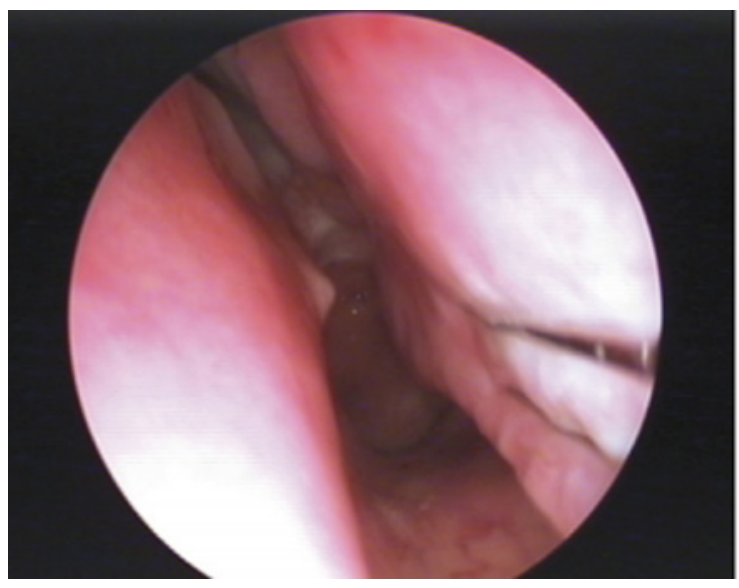

Un aspecto positivo de este procedimiento es el hecho que se puede realizar como un procedimiento extrahospitalario, solo con anestesia local (Schumacher, 2004). El tratamiento comienza con un anestésico tópico aplicado al cornete inferior durante 1-5 minutos, seguido de una inyección anestésica local. El electrodo de aguja de RF se inserta por vía submucosa en 1 a 4 sitios diferentes del cornete inferior hasta el cornete óseo (Figura 3). Es importante no dañar la mucosa posterior al sitio de punción. Después de este procedimiento, no hay necesidad de tratamiento local, solo tiene que presionar durante unos minutos con un algodón.

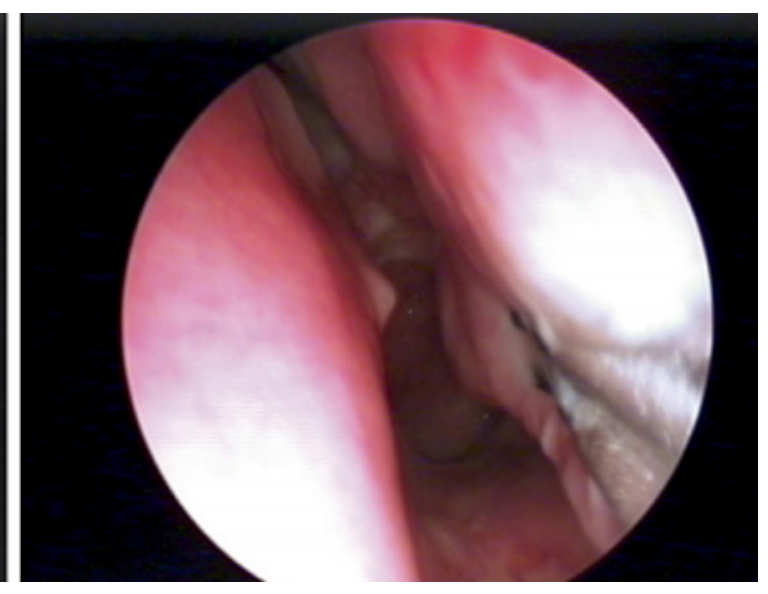

Figura 3. Reducción de la mucosa del cornete inferior con radiofrecuencia: vista intraoperatoria

Fuente: (Schumacher, 2004)

\section{Reducción de turbinado por ultrasonido}

Este método es eficiente para la reducción de los cornetes nasales agrandados, porque

los tejidos afectados están expuestos a la desintegración ultrasónica, por medio de una fluctuación submucosa de baja frecuencia de una sonda nasal ultrasónica. Esto resulta en la destrucción de los tejidos cavernosos y de conexión, con la posterior reducción del volumen de cornetes como resultado de cicatrices infraturbinadas. (Hol \& Huizing, 2016)
Después de esta intervención, se observó disminución de la secreción de moco y se observó edema intersticial, presencia profusa de haces de fibroblastos y fibras de colágeno, epitelio estratificado de células columnares y escasa copas de células evidentes, combinadas con islas de metaplásticos epitelio.

Otras técnicas quirúrgicas menos utilizadas son: quimiocauterización, criocirugía, neurectomía vidiana. Cuando la crioterapia se usa para el tratamiento de la rinitis vasomotora crónica, se obtienen mejores resultados con una mayor duración de congelación y también mayor área de administración. 


\section{Conclusiones}

Durante el desarrollo de la investigación se observó que la reducción del cornete inferior es simple y poco invasiva para abordar la obstrucción nasal secundaria a los cornetes inferiores agrandados. Someterse a algún tipo de operación quirúrgica no es un procedimiento complejo y se puede hacer bajo anestesia local con un mínimo de morbilidad postoperatoria, dejando en evidencia que esta operación tiene el propósito de mejorar la calidad de vida, así como también, mejorar la longevidad y reducir el riesgo de morbilidad médica.

\section{Bibliografía}

Archer, S., \& Meyers, M. (2019). Turbinate Dysfunction. Nueva York, Estados Unidos: Medscape. Recuperado el 29 de Enero de 2020

Bolívar, J. (2015). Investigación Documental. México: Pax.

Castro, J. (2016). Técnicas Documentales. México: Limusa.

Chhabra, N. (2009). The diagnosis and management of empty nose syndrome. Otolaryngologic Clinics of North America, 42(2), 311-30.

Davila, A. (2015). Diccionario de Términos Científicos. Caracas: Editorial Oasis.

Elwany, S., \& Abel, S. (2009). Laser surgery for allergic rhinitis: the effect on seromucinous glands. Otolaryngol Head Neck Surg.

Farmer, \& Eccles. (2012). Agrandamiento crónico del cornete inferior y las implicaciones para la intervención quirúrgica. Rinología, 234-238.

Fradis, M., Golz, A., \& Danino, J. (2011). Inferior turbinectomy versus submucosal diathermy for inferior turbinate hypertrophy. Ann Otol Rhinol Laryngol.

Gindros, \& Kantas. (2009). Mucosal changes in chronic hypertrophic rhinitis after surgical turbinate reduction. Eur Arch Otorhinolaryngol, 266:14091416 .

Goode, R. (2010). Surgery of the turbinates. . J Otolaryngol.

Hol, M., \& Huizing, E. (2016). Treatment of inferior turbinate pathology: a review and critical evaluation of the different techniques. Rhinology, 157-66.
Antes de cualquier tipo de intervención, el cirujano tiene que ser familiarizado con la justificación y las indicaciones para cada técnica de cirugía Una revisión sistemática del paciente ayudará en la identificación de regiones de obstrucción y minimizará el riesgo de posibles complicaciones. El objetivo de esta intervención es mantener el flujo de aire nasal y la permeabilidad para reducir la respiración bucal. No existe un procedimiento único que pueda aplicarse a todos los pacientes. Por esta razón, es muy importante que el cirujano conozca una gran variedad de técnicas para elegir el correcto para un específico paciente.

Jones, A. (2013). Predecir el resultado de la diatermia submucosa a los cornetes inferiores. Clin Otolaryngol.

Leong, \& Eccles, R. (2015). Cirugía de cornete inferior y flujo de aire nasal: manejo basado en evidencia. . Curr Opin Otolaryngol Head Neck Surg., 18 (1): 54-9.

Mucci S., S. A. (2017). Turbinectomía parcial inferior: un procedimiento eficaz para la rinitis crónica. Ear Nose Throath J., 405-457.

Ophir D., S. A. (2015). Total inferior turbinectomy for nasal airway obstruction. Arch Otolaryngol Head Neck Surg.,

Ozcan, K., Gedikli, Y., Ozcan, I., Pasaoglu, L., \& Dere, H. (2008). Microdebrider for reduction of inferior turbinate: evaluation of effectiveness by computed tomography. . J Otolaryngol Head Neck Surg.

Passali, D. (2013). Rhinosinusal Inflammation And Infections: Modern Thinking And Current Treatment. . Bucharest: Ed. Academiei Romane.

Schumacher, M. (2004). Nasal dyspnea: the place of rhinomanometry in its objective assessment. Am J Rhinol., 18(1):41-6.

Steiner W., W. A. (2006.). Lasers in otorhinolaryngology, head and neck surgery. Tuttlingen: Endo-Press.

Zapanta, P. (2014). Turbinectomía. Recuperado el 29 de Enero de 2020, de Medscape: http://emedicine.medscape.com/article/2051775-overview 


\section{CITAR ESTE ARTICULO:}

Parrales Vidal, I., Reyes Tigrero, K., Crespo Zamora, M., \& Chavez Reyes, J. (2020). Turbinectomia en hipertrofia de cornetes nasales. RECIAMUC, 4(1), 29-39. doi:10.26820/reciamuc/4.(1).enero.2020.29-39

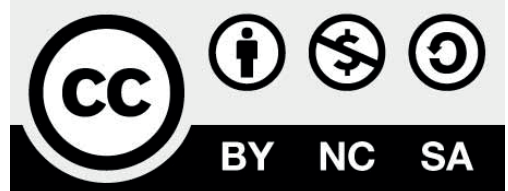

RECONOCIMIENTO-NOCOMERCIAL-COMPARTIRIGUAL CC BY-NC-SA

ESTA LICENCIA PERMITE A OTROS ENTREMEZCLAR, AJUSTAR Y CONSTRUIR A PARTIR DE SU OBRA CON FINES NO COMERCIALES, SIEMPRE Y CUANDO LE RECONOZCAN LA AUTORÍA Y SUS NUEVAS CREACIONES ESTÉN BAJO UNA LICENCIA CON LOS MISMOS TÉRMINOS. 